\title{
Keyword Index Volume 7 (1999)
}

\begin{tabular}{|c|c|c|}
\hline antimicrobial activity & 359 & hand grasp \\
\hline aspiration & 359 & $\begin{array}{l}\text { hemodynamics (G9.330.612.+) } \\
\text { higher-order statistics and spectra }\end{array}$ \\
\hline biofluid & 271 & hyperemia \\
\hline biomedical instrumentation & 143 & \\
\hline biosignal & 137 & ICU patient \\
\hline bispectral analysis & 85 & infants \\
\hline bladder & 393 & information systems \\
\hline blood & 171 & isoflurane \\
\hline blood circulation (G9.330.163) & 225 & \\
\hline blood flow & 205,219 & Kalman filter \\
\hline blood flow signal & 185 & \\
\hline blood perfusion & 125 & laser-Doppler \\
\hline blood supply & 143 & laser-Doppler flowmetry \\
\hline breath analysis & 29 & $\begin{array}{l}\text { laser-Doppler flux } \\
\text { laser-Doppler technique }\end{array}$ \\
\hline cerebral hemoglobin concentration & 63 & \\
\hline chaos & 205 & mass spectrometry \\
\hline characteristic curve & 359 & mathematics - algorithms (H1.548.35) \\
\hline classification & 85 & mechanical ventilation \\
\hline coherence & 103 & $\begin{array}{l}\text { microaspiration } \\
\text { microcirculation }\end{array}$ \\
\hline data compression & 285 & microperfusion \\
\hline database design & 331 & microsurgery \\
\hline decontamination & 359 & monitoring \\
\hline delay time & 243 & Monte Carlo \\
\hline dialysis & 371 & MTF \\
\hline diffusion capacity & 271 & mucus \\
\hline digital signal filtering & 125 & multivariate time-varying autoregression \\
\hline disturbance & 185 & \\
\hline Doppler shift & 143 & nasal physiology \\
\hline Doppler spectrum & 185 & natural sensors \\
\hline drop-foot & 393 & $\begin{array}{l}\text { near infrared spectrophotometry } \\
\text { nerve cuff electrode }\end{array}$ \\
\hline EEG & 285 & neural nets \\
\hline electronic patient record & 331 & nifedipine \\
\hline endotracheal tube & 359 & $\begin{array}{l}\text { nifedipine (D3.383.725.203.540) } \\
\text { nonlinear dynamics }\end{array}$ \\
\hline FES & 393 & nonlinear dynamics (H1.548.675) \\
\hline flowmetry & 171 & \\
\hline flowmotion patterns & 113 & optical methods \\
\hline fractal & 205 & osmotic resistance \\
\hline FRC & 243 & \\
\hline free flap & 219 & perfusion \\
\hline frequency analysis & 219 & $\begin{array}{l}\text { phosphors } \\
\text { platelets }\end{array}$ \\
\hline gas exchange & 243 & polysomnography \\
\hline gas transport & 243 & positioning \\
\hline gas viscosity & 243 & preterm \\
\hline
\end{tabular}

$163,171,185,193$

$85,103,125,143$ 
principal component analysis principal components

probe

pulmonary gas exchange

Raynaud's disease

Raynaud's disease (C14.907.744.+)

Raynaud's phenomenon

sampling capillary

SCET

silver

silver-coated endotracheal tube

silver-coating

simulations

sleep disorders

Keyword Index Volume 7 (1999)

285

137

63

271

193

225

$85,103,113,163$

243

359

359

359

359

171

331 spectral analysis

spectral estimation

subband coding

tissue

tissue motion

vasodilation

vasomotion

vector quantization

velocity spectrum

ventilator-associated pneumonia

ventilatory support

$\mathrm{X}$-ray image detectors

$\mathrm{X}$-ray luminescence
$103,113,163$

125

285

171

185

113

219

285

185

359

243

\section{3}

53 